\title{
Tröhler, Daniel
}

\section{Wissenschaftliche Ehrungen oder der doppelte Gestus der Auszeichnung. Bemerkungen zur fallengelassenen Idee eines Heinrich Roth-Forschungspreises der DGfE}

Erziehungswissenschaft 25 (2014) 49, S. 43-46

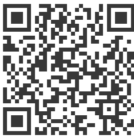

Quellenangabe/ Reference:

Tröhler, Daniel: Wissenschaftliche Ehrungen oder der doppelte Gestus der Auszeichnung.

Bemerkungen zur fallengelassenen Idee eines Heinrich Roth-Forschungspreises der DGfE - In:

Erziehungswissenschaft 25 (2014) 49, S. 43-46 - URN: urn:nbn:de:0111-pedocs-100795 - DOI:

10.25656/01:10079

https://nbn-resolving.org/urn:nbn:de:0111-pedocs-100795

https://doi.org/10.25656/01:10079

in Kooperation mit / in cooperation with:

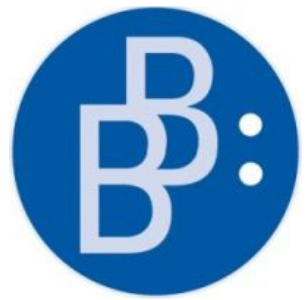

https://www.budrich.de

\section{Nutzungsbedingungen}

Gewährt wird ein nicht exklusives, nicht übertragbares, persönliches und beschränktes Recht auf Nutzung dieses Dokuments. Dieses Dokument ist ausschließlich für den persönlichen, nicht-kommerziellen Gebrauch bestimmt. Die Nutzung stellt keine Übertragung des Eigentumsrechts an diesem Dokument dar und gilt vorbehaltlich der folgenden Einschränkungen Auf sämtlichen Kopien dieses Dokuments müssen alle Urheberrechtshinweise und sonstigen Hinweise auf gesetzlichen Schutz beibehalten werden. Sie dürfen dieses Dokument nicht in irgendeiner Weise abändern, noch dürfen Sie dieses Dokument für öffentliche oder kommerzielle Zwecke vervielfältigen, öffentlich ausstellen, aufführen, vertreiben oder anderweitig nutzen.

Mit der Verwendung dieses Dokuments erkennen Sie die Nutzungsbedingungen an.

\section{Terms of use}

We grant a non-exclusive, non-transferable, individual and limited right to using this document.

This document is solely intended for your personal, non-commercial use. Use of this document does not include any transfer of property rights and it is conditional to the following limitations: All of the copies of this documents must retain all copyright information and other information regarding legal protection. You are not allowed to alter this document in any way, to copy it for public or commercial purposes, to exhibit the document in public, to perform, distribute or otherwise use the document in public.

By using this particular document, you accept the above-stated conditions of use.

\section{Kontakt / Contact:}

peDOCS

DIPF | Leibniz-Institut für Bildungsforschung und Bildungsinformation Informationszentrum (IZ) Bildung

E-Mail: pedocs@dipf.de

Internet: www.pedocs.de

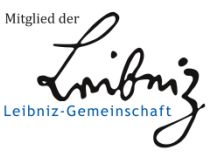




\section{Erziehungswissenschaft}

Mitteilungen der Deutschen Gesellschaft

für Erziehungswissenschaft (DGfE)

Heft 49

25. Jahrgang 2014

ISSN 0938-5363

Verlag Barbara Budrich 


\section{INHALTSVERZEICHNIS}

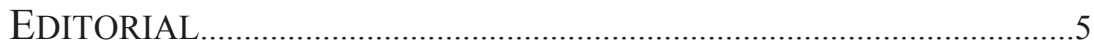

BEITRÄGE ZUM THEMA ,ERINNERUNGSKULTUREN PREISE, NAMEN UND DISZIPLINPOLITIK“

Stellungnahme des Vorstandes zur Rücknahme des Namens für den

Forschungspreis vom 13. Januar 2014

Andreas Hoffmann-Ocon

Heinrich Roth und der Preis der Forschung - Historisierungen,

Verundeutlichungen und erziehungswissenschaftliche

Erinnerungsgeschichten

Benjamin Hasselhorn

Erinnerung im Streit. Zum Umgang der deutschen

Geschichtswissenschaft mit ihrer eigenen Vergangenheit

Eva Matthes \& Carola Groppe

Historische Bildungsforschung und Erinnerungskultur. Eine

Stellungnahme der Sektion Historische Bildungsforschung.

Daniel Tröhler

Wissenschaftliche Ehrungen oder der doppelte Gestus der

Auszeichnung. Bemerkungen zur fallengelassenen Idee eines Heinrich

Roth-Forschungspreises der DGfE

Jörg Schlömerkemper

„Nomen est omen“ - oder geht es auch ohne? Was kann/Was soll der

Name einer Person bei einem Preis bedeuten? Anmerkungen nach dem

Streit um die Benennung des Forschungspreises der DGfE nach

Heinrich Roth

BEITRÄGE

Adolf Kell

Grenzgänge, Traditionen und Zukünfte in der Deutschen Gesellschaft für Erziehungswissenschaft. Kongresse zur Reflexion - auch für die Sektion Berufs- und Wirtschaftspädagogik? 


\section{MitTEILUNGEN DES VORSTANDS}

Protokoll der Mitgliederversammlung der Deutschen Gesellschaft für

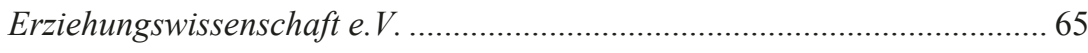

Ergebnis der Wahl zum Vorsitz und Vorstand der DGfE............................ 71

Liste der unbekannt verzogenen Mitglieder ............................................... 72

BERICHTE AUS DEN SEKTIONEN

Sektion 1 - Historische Bildungsforschung ............................................... 75

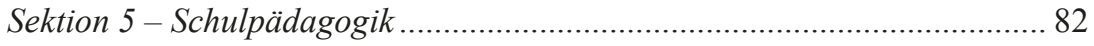

Sektion 8 - Sozialpädagogik und Pädagogik der frühen Kindheit................ 85

Sektion 9 - Erwachsenenbildung .............................................................. 91

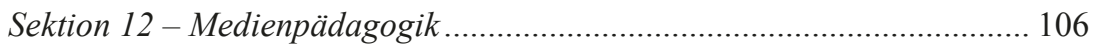

Sektion 13 - Differentielle Erziehungs- und Bildungsforschung ................ 108

NOTIZEN

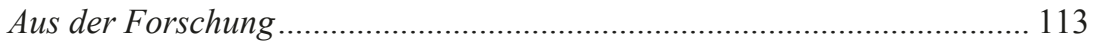

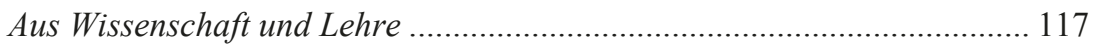

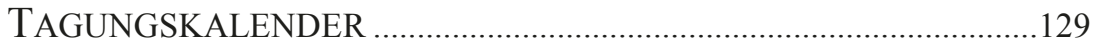




\title{
Wissenschaftliche Ehrungen oder der doppelte Gestus der Auszeichnung
}

\author{
Bemerkungen zur fallengelassenen Idee eines Heinrich Roth- \\ Forschungspreises der DGfE
}

\section{Daniel Tröhler}

Mit einem Heinrich Roth-Preis wollte die Deutsche Gesellschaft für Erziehungswissenschaft (DGfE) 2014 erstmals einen Forschungspreis verleihen. Wegen des gewählten Stiftungs-Namens gab es Proteste, Anschuldigungen, Erklärungen, und Eckhard Klieme, der Preisträger, wurde schließlich anlässlich des DGfE-Kongresses 2014 der Forschungspreis der DGfE übergeben ohne Heinrich Roth im Titel des Preises.

Wie war es dazu gekommen? Zunächst stellen sich mehrere Fragen: Wer schlug Heinrich Roth als besondere Auszeichnung des Forschungspreises vor, wer hat ihn unter einer Auswahl von möglichen weiteren ehrenvollen Namen ausgewählt, und was waren die Kriterien? Und wie kam die Wahl des Preisträgers zu Stande, wer hat die Kriterien bestimmt, wer mögliche Kandidatinnen und Kandidaten für diesen Preis ausgewählt und wer die Evaluationen durchgeführt? Das alles war, nicht ganz unverständlich, nie Gegenstand der zum Teil heftig geführten Diskussionen.

Gegenstand dieser Diskussionen war, dass der Forschungspreis den Namen einer Person mit durchaus zweifelhafter Vergangenheit trug: Heinrich Roth, geboren 1906 in Gerstetten (Württemberg), ehemaliger Wandervogel, dann Lehrer und anschließend Student, unter anderem bei begeisterten Nationalsozialisten, dann dem Regime mindestens angepasster Heerespsychologe, Soldat, später Lehrer an der Hochschule für internationale Pädagogische Forschung HIPF, der Vorgängerinstitution des DIPF, dessen späterer Direktor, Eckhard Klieme, nun eben diesen Heinrich Roth-Forschungspreis der DGfE 2014 erhalten sollte. Die Brücke zwischen Roth und Klieme lässt sich schlagen, sollte doch Roths Tätigkeit nach 1960 so etwas wie eine (allerdings sehr verhaltene) „realistische Wendung“ der deutschen Pädagogik einläuten und damit der Pädagogik den Weg zur Erziehungswissenschaft ebnen, in deren Tradition Klieme zu stehen scheint.

Die geplante Preisverleihung war keine sehr gute Idee. Nicht die Wahl Eckhardt Kliemes zum Preisträger, aber jene von Heinrich Roth als Namensgeber des Forschungspreises, trotz ihrer jeweiligen Sympathie zur empirischen Bildungsforschung und trotz ihrer gemeinsamen HIPF/DIPF-Zugehörigkeit. Angesichts der Biographie Roths trat Empörung auf, von Besorgten, Moralisten und vermutlich auch von Heuchlern, wer weiß, und der Vorstand 
der DGfE sah sich genötigt, nicht auf den Forschungspreis zu verzichten, sondern schnellstens auf den Namen Heinrich Roth. Er wählte nunmehr den Weg der Transparenz und informierte, nicht ohne ein (berechtigtes) Plädoyer für eine differenzierte Betrachtungsweise zu halten und zu schließen:

„Ein Traditionen aufgreifender und gleichzeitig Identifikationen ermöglichender Name für einen Forschungspreis, wie es hier bezweckt werden sollte, ist der Heinrich Roths allerdings nicht. Und möglicherweise ist der Versuch, eine solche Traditionsstiftung mit einem Namen zu erreichen, nicht aussichtsreich und vielleicht auch gar nicht sinnvoll.“ (Vorstand der DGfE 2014: 10)

Sicher ist: Hätten wir einen Immanuel Kant, der in eine - unsere - Traditionslinie hätte eingepasst werden können, wäre die Diskussion wohl gar nicht entbrannt: Der Geehrte wäre geehrt und der Vorstand der DGfE zufrieden gewesen. Aber Kant gehört nicht zu ,uns', trotz seiner in Nachschrift veröffentlichten Vorlesung über Pädagogik, die zudem nun ja wirklich kein Glanzstück der Pädagogik ist. Aber wen sollte man dann zur Symbolfigur für einen Forschungspreis wählen? Kants zeitweiliger Held Jean-Jacques Rousseau, der Genderforscherinnen auf den Plan hätte rufen können? Davon abgesehen war er Genfer und hätte damit nicht so richtig in die akademische Tradition Deutschlands gepasst. Ein Pestalozzi-Forschungspreis für deutsche Erziehungswissenschaften 2014? Aber auch er war bekanntlich kein Deutscher und auch nicht so richtig Wissenschaftler, weil institutionell nicht akademisch eingebunden.

Besser passen würde dann schon ein späterer Bewunderer Pestalozzis, Ernst Christian Trapp, erster Professor für Pädagogik in Halle, vier Jahre nach seiner Berufung allerdings wieder vertrieben. Doch es gibt schon einen DGfE-Preis, der nach ihm benannt wurde, der „Ernst-Christian-Trapp-Preis der Deutschen Gesellschaft für Erziehungswissenschaft“, der für ,,innovative und unkonventionelle wissenschaftliche Leistungen im Fach Erziehungswissenschaft" (DGfE 2014) vergeben wird. Ob das nun heißt, dass der neu eingeführte Forschungspreis, der an Eckhard Klieme vergeben wurde, Innovation und Originalität ausschließt oder ob ,innovative und unkonventionelle wissenschaftliche Leistungen" nicht als Forschung verstanden werden, muss offen bleiben. Demgegenüber steht allerdings die Laudatio, die von den ,innovativen wissenschaftlichen Forschungsleistungen von Eckhard Klieme im Bereich der empirischen Erfassung von Unterrichtsqualität, Unterrichtskulturen und schulischen Leistungen sowie von seinem Engagement auf dem Gebiet der pädagogischen Diagnostik“" (Benner/Hascher/Thole 2014: 37) spricht. Warum man dann Eckhard Klieme nicht den „Ernst-Christian-TrappPreis der Deutschen Gesellschaft für Erziehungswissenschaft" verlieh, ist eine weitere Frage. Sie führt unmittelbar zur nächsten:

Die vermutlich entscheidende Frage für diese ganze Geschichte richtet sich auf die mögliche Motivation des neuen Preises. Bekannt ist, dass 2012 
im Vorfeld der Einrichtung des neuen DGfE-Preises unter anderem auch einige DGfE-Mitglieder die Gesellschaft für Empirische Bildungsforschung (GEBF) gründeten, mit dem Ziel, die empirische Bildungsforschung verstärkt $\mathrm{zu}$ fördern und deren Ergebnisse besser zu verbreiten. Eine Sezession von Mitgliedern ist aber für keinen Vorstand einer Gesellschaft ein Kompliment, weshalb sich eine neu geschaffene Auszeichnung für eines der prominenten Aushängeschilder der empirischen Bildungsforschung geradezu aufdrängte: Signale, die Neuland versprechen, sollen klar und deutlich sein.

Oder doch lieber nicht allzu klare? Dass man diesen neuen Preis mit einem Wegbereiter der empirischen Bildungsforschung adeln wollte, macht aus diesem Blickwinkel durchaus Sinn. Das gehört zu den strategischen Entscheidungen als Teil der Vorstandsarbeit, wobei auch mal ein Fauxpas passieren kann, wie mit Heinrich Roth. Wäre in diesem Zusammenhang eigentlich, ganz nebenbei gefragt, nicht vielleicht Saul B. Robinsohn besser geeignet gewesen? Mutiger allemal, nicht zuletzt weil Robinsohn weit stärker als Roth - der ja noch weitgehend in der nicht ganz lupenreinen geisteswissenschaftlichen Pädagogik verhaftet war - nach seiner Remigration eine neue, international orientierte Erziehungswissenschaft und Bildungspolitik anstrebte. $\mathrm{Zu}$ viel Neuland sollte es dann doch nicht sein, weil es ja auch noch um Tradition ging, in die nun Robinsohn weit schlechter passt als Roth. Und das ist das eigentliche Problem.

Es ist wichtig und richtig, dass sich Vorstände darüber Gedanken machen, wie das Sozialleben ihrer Gesellschaft am besten am Leben zu erhalten sei. Dazu gehören Periodika und Tagungen, gelegentlich (und in Deutschland besonders häufig, wie mir scheint) öffentliche Stellungnahmen und - eben Ehrungen. Das alles ist legitim und mit organisationssoziologischen Studien wohl auch genügend begründbar. Ob allerdings die ,Stiftung' von Traditionen zu dieser Ehrung gehört, ist mehr als fraglich und dies gleich aus verschiedenen Gründen, die alle auf unterschiedliche Aspekte dessen verweisen, was ich als den doppelten Gestus der Auszeichnung bezeichne:

- Traditionen kann man nicht erfinden, sondern man steckt in ihnen, und will man im Namen der Tradition jemanden wie Klieme ehren, so ehrt man auch die Tradition. Das muss nicht nur im Falle Deutschlands sehr gut überlegt sein.

- Die Kanonisierung der Tradition durch die Auswahl von Stiftungsexponenten (Trapp, Roth) ist eine Bemächtigung, gegen die sie sich diese Exponenten gar nicht mehr wehren können und die als Inklusion auch Exklusion (zum Beispiel Robinsohn) in Kauf nimmt. Auch das muss nicht nur in Deutschland sehr gut überlegt sein.

- Die Bemächtigung der Tradition als besondere Ehrung des zu Ehrenden ist ein kollektivistischer Akt, der die besondere Leistung des zu Ehrenden relativiert, gerade wenn es um Innovation geht. Wie viel Innovation darf es denn sein? Und auch das gilt nicht nur in Deutschland. 
Tradition und Geschichtsschreibung sind heute nicht mehr identisch und vermutlich sind sie sogar irgendwie Feinde, was im 19. Jahrhundert noch übersehen wurde, weil erstere (offenbar) in all ihrer Macht gewissermaßen subkutan wirkt, während letztere sich methodisch - historiographisch und komparativ - gegen diese Macht stemmen muss, ohne sie ignorieren zu können. Eine wissenschaftliche Ehrung im Sinne der Tradition kann daher nur irgendwie schief gehen. Die Zusammensetzung des Vorstandes der DGfE hätte eigentlich genügend Expertise erwarten lassen können, sich diesem Machtspiel zu entziehen und Eckhard Klieme schon im ersten Anlauf den (Forschungs-) Preis zu verleihen, den der Vorstand ihm dann auch wirklich verliehen hat, nachdem er in der Folge des Protestes gegen die Idee eines Heinrich RothPreises Heinrich Roth aus dem Titel hatte fallen lassen.

$\mathrm{Ob}$ der Vorstand der DGfE mit seiner Strategie des neuen Preises Erfolg haben wird? Die Frage wird nicht damit entschieden, ob DGfE-Mitglieder jetzt oder später in die Gesellschaft für Empirische Bildungsforschung (GEBF) eintreten und sogar noch der DGfE den Rücken drehen (Austritt), sondern ob es der DGfE gelingt, sich mit den Mitteln der Wissenschaft der unreflektierten Macht der Tradition zu entziehen. Dieses Desiderat wurde mal, in anderen Kontexten und moralisch auf- und überladen, Emanzipation genannt, und sollte heute eine Selbstverständlichkeit sein, die nicht nur Deutschland mit seiner zweifellos schwierigen Geschichte betrifft, sondern moderne wissenschaftliche Forschung insgesamt und überall. Es müsste eigentlich zum Standard geworden sein. Hätte sich dieser Standard früher durchgesetzt, hätte sich womöglich die ganze Affäre gar nie ereignet.

Daniel Tröhler, Prof. Dr., ist Hochschullehrer für Erziehungswissenschaft an der Universität Luxemburg.

\section{Literatur}

Benner, Dietrich/Hascher, Tina/Thole, Werner (2014): Laudatio für Prof. Dr. Eckhard Klieme anlässlich der Verleihung des DGfE-Forschungspreises 2014. In: Erziehungswissenschaft 25, 48, S. 37-41.

Deutsche Gesellschaft für Erziehungswissenschaft (DGfE) (2014): ErnstChristian-Trapp-Preis. www.dgfe.de/wir-ueber-uns/ernst-christian-trapppreis.html [Zugriff: 05. November 2014].

Vorstand der Deutschen Gesellschaft für Erziehungswissenschaft (DGfE) (2014): Stellungnahme des Vorstandes zur Rücknahme des Namens für den Forschungspreis vom 13. Januar 2014. In: Erziehungswissenschaft 25,48 , S. 9-10. 